\title{
Towards single biomolecule handling and characterization by MEMS
}

\author{
Hideyuki F. Arata $\cdot$ Momoko Kumemura • \\ Naoyoshi Sakaki $\cdot$ Hiroyuki Fujita
}

Received: 26 November 2007 / Revised: 25 December 2007 / Accepted: 8 January 2008 /Published online: 25 March 2008

(C) Springer-Verlag 2008

\begin{abstract}
Applications of microelectromechanical systems (MEMS) technology are widespread in both industrial and research fields providing miniaturized smart tools. In this review, we focus on MEMS applications aiming at manipulations and characterization of biomaterials at the single molecule level. Four topics are discussed in detail to show the advantages and impact of MEMS tools for biomolecular manipulations. They include the microthermodevice for rapid temperature alternation in real-time microscopic observation, a microchannel with microelectrodes for isolating and immobilizing a DNA molecule, and microtweezers to manipulate a bundle of DNA molecules directly for analyzing its conductivity. The feasibilities of each device have been shown by conducting specific biological experiments. Therefore, the development of MEMS devices for single molecule analysis holds promise to overcome the disadvantages of the conventional technique for biological experiments and acts as a powerful strategy in molecular biology.
\end{abstract}

Keywords Microelectromechanical systems. Single molecule $\cdot$ Microheater $\cdot$ Microcontainer .

Microtweezers $\cdot$ Microchannel

\section{Introduction}

Miniaturized microdevices have various advantages in terms of functions compared with their macroscopic counterparts:

H. F. Arata $(\bowtie) \cdot$ M. Kumemura $\cdot$ N. Sakaki $\cdot$ H. Fujita Institute of Industrial Science (IIS), The University of Tokyo, 4-6-1, Komaba, Meguro-ku,

Tokyo 153-8505, Japan

e-mail: arata@iiis.u-tokyo.ac.jp higher sensitivities in sensors, high efficiencies in reactors, small amount of samples required, fast response, etc. Microelectromechanical systems (MEMS) technologies have been developed by applying semiconductor microfabrication technologies from the late 1980s. MEMS technologies offer the advantages of the batch fabrication of many devices and have the ability to integrate multiple functional units in a small area as well as the ability to fabricate small structures at the submicrometer level. MEMS technologies have successfully been applied to sensors [1, 2], actuators [3], microreactors [4, 5], radiofrequency devices [6], and optical devices such as an optical attenuator [7], an optical mirror array [8], an optical scanner array [9], and optical switches [10-12]. Besides the application of MEMS to industrial products such as accelerometers and movable mirror arrays for a video projection system, applications to biological and biochemical experiments have become a hot topic. MEMS technologies have also enabled the integration of multiple-function units on a small chip, so-called micro total analysis systems ( $\mu$ TAS) or lab on a chip, whose basic idea was proposed by Manz et al. [13] in 1990. $\mu$ TAS allow rapid biological or chemical analysis with a small amount of sample thanks to the merits of miniaturization. While the popular industrial application is limited to DNA chips, there has been an increase both in the amount of research aimed at biological and biochemical applications and in the number of applications [14, 15].

Single molecule observation and characterization of biomolecules enables us to determine the individual behavior and characteristics of molecules usually hidden in the ensemble-averaging and time-averaging of bulk experiments $[16,17]$. The major advantages in single molecule characterization are the ability to measure the variations in molecules, the dynamics on a short time scale, and the mechanical motion of biomolecules. The typical size of an individual biomolecule is on the order of a few 
nanometers to tens of nanometers. Giant nucleic acids such as a $\lambda$ DNA are longer than $15 \mu \mathrm{m}$. Today, we can fabricate microstructures less than $100 \mathrm{~nm}$ in size by MEMS technologies. Because their scales have become closer, developing MEMS devices to manipulate a single biomolecule might realize novel experiments or measurements with higher sensitivity that could not have been achieved by a conventional single molecule manipulation technique such as using magnetic tweezers [18, 19], optical tweezers $[20,21]$, atomic force microscope cantilevers [22], and liquid flow [23]. Some attempts to manipulate a single biomolecule with microstructures or microfabricated devices have been reported. Controlling and on-off switching of actomyosin as a nanoactuator [24] and electrostatic manipulation of DNA in microfabricated structures $[25,26]$ were successfully achieved. However, these works did not utilize the full ability of MEMS technologies such as high sensitivity or manipulation of an individual molecule.

This review is aimed at introducing some new achievements in MEMS application to single biomolecule manipulation and characterization by our group. The target biomolecules are proteins (a motor protein $\mathrm{F}_{1}$-ATPasehereafter $F_{1}$ - and an enzyme $\beta$-galactosidase - hereafter $\beta$ gal) and DNAs. Although many other exciting results exist, four examples are highlighted owing to the limited scope of this review. The first is a microthermodevice for a real-time rapid temperature alternation experiment whose capability was demonstrated by controlling a rotary biomolecular motor, $F_{1}$ [27]. The rapid control of the temperature enabled us to perform rapid motion control of $F_{1}$. The experimental results suggest that the torque of $F_{1}$ increases at higher temperatures. The second example is the application of a femtoliter container whose temperature can be controlled rapidly by a microthermodevice [28]. The catalytic reaction of an enzyme, $\beta$-gal, was observed in the microcontainer with rapid temperature alternation. This method realized the enzymatic assay at temperatures higher than that at which enzymes would "normally" be denatured in bulk experiments. The third example is a systematic isolation and immobilization of a single DNA molecule with a microdevice [29]. It consists of a polymer sheet with channels and aluminum electrodes deposited on a cover slip. A DNA molecule passed through a $2-\mu \mathrm{m}$-wide channel and both ends of a single DNA molecule were immobilized at integrated electrodes by dielectrophoresis force. The fourth example is the conductivity measurement of DNA bundles; this measurement demonstrates that humidity is the main factor affecting the electrical conduction in a DNA bundle captured between microactuated silicon tweezers [30]. The result suggests that (1) DNA has a quasi-ohmic behavior under constant temperature and humidity conditions and (2) the exponential dependence of conductivity on the relative humidity could be explained by the change of permittivity arising from water adsorption on the DNA backbone. A quantitative model was also proposed to explain the electrical properties of the DNA bundle. For more detailed information, especially on the experimental conditions and fabrication processes, we encourage readers to refer to the original papers. The significance of each microdevice for its biological application will be discussed specifically for each topic. In the conclusion, the general advantages and the future prospects of MEMS devices for the single biomolecule experiments will be discussed.

\section{Motion control of a rotary biomolecular motor by a microfabricated local heating device}

Motor proteins [31] are the major targets in single molecule observation because their mechanical motions allow measurement of the energy consumption of a single molecule, which is important information for understanding molecular mechanisms. Enzymes such as kinesin [32], dynein [33], myosin [34], DNA helicase [35], and RNA polymerase [36] act as a linear motor which moves on the rail proteins or DNA. Bacterial flagella protein complex [37] and a subunit of ATP synthase, $F_{1}$ ) [17, 38-41], act as a rotary motor. Changing the chemical conditions in bioexperiments cannot realize real-time rapid alternation in experimental conditions [42-44]. One of the most promising ways to measure the response of an individual molecule is rapid temperature alternation because the activity of proteins is known to be temperature dependent from bulk experiments $[45,46]$. In this work, Arata et al. proposed a simple and versatile method to control the motion and activity of a single biomolecular motor by rapid temperature alternation.

Conventional devices which control the temperature under a microscope have the following limitations: (1) the response speed is low (on a time scale of minutes), (2) thermal expansion of the stage plate of the microscope causes defocusing during temperature alternation, and (3) the heat causes damage to the objective lenses. These limitations can be overcome by controlling the temperature locally $[47,48]$ because reducing the volume of the heating target reduces the total amount of heat generated to alter the temperature. Local heating with an infrared laser may damage proteins by exposing the samples to excess energy though it can alter the temperature rapidly in a local area [48]. A microfabricated local heating device can provide a good solution by integrating a microheater and a microthermosensor on a glass plate. The local heating microdevice was fabricated by patterning nickel on the glass plate (Fig. 1a). The microheater was designed with a circular shape so as to minimize the temperature nonuniformity within the heater. The diameter of the circular microheater was $400 \mu \mathrm{m}$, and the width was $20 \mu \mathrm{m}$. The maximum 
Fig. 1 a Ni-patterned glass plate, which is placed on a pitch conversion board and connected to lead wires (left). Microscope view of an integrated microheater and microthermoresistive sensor (right). Reducing the size of the thermodevice can reduce the total amount of heat generated to alter the temperature. This enables us to avoid drawbacks in conventional devices and realizes high response speed. b Microscope view of two microbeads being rotated by a single $\mathrm{F}_{1}$-ATPase $\left(F_{1}\right)$ molecule. The angular velocity is $1.5 \mathrm{rps}$. The time course of the rotation of $F_{1}$ while alternating the temperature. The angular velocity changed from 1.2 to $4.4 \mathrm{rps}$ and back to 1.7 rps. (Reprinted with permission from Arata et al. [27]. Copyright 2006, American Institute of Physics.) a

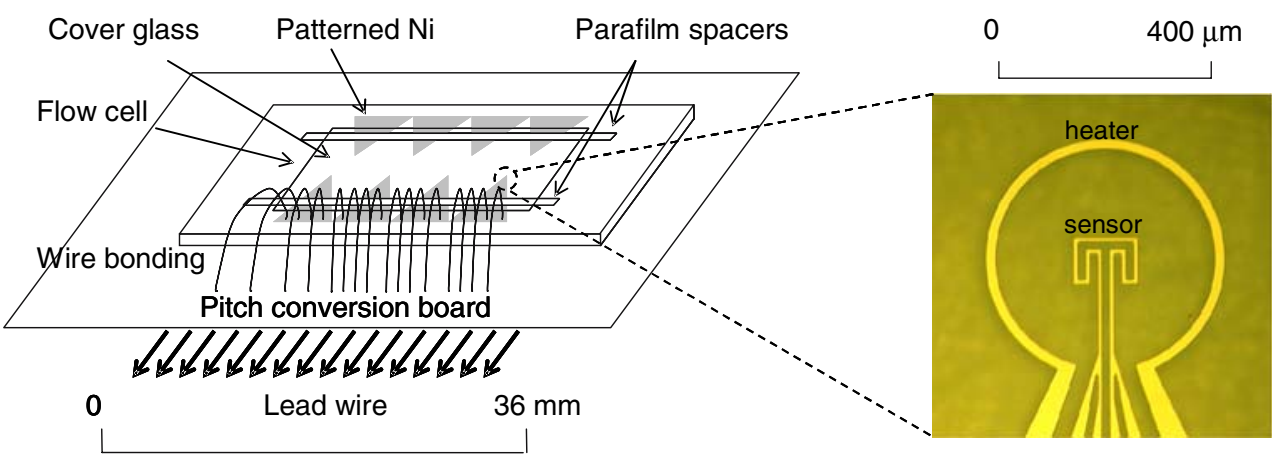

b

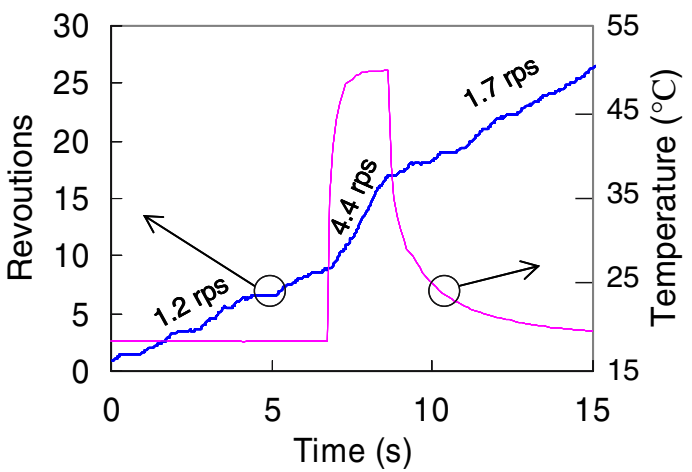

response speed was 71.5 and $56.9{ }^{\circ} \mathrm{C} / \mathrm{s}$ for temperature rise and fall, respectively. The microthermosensor was located at the center of the microheater to realize real-time monitoring of the temperature. The temperature distribution in water was measured with high spatial resolution using the fluorescent dye rhodamine B. Since rhodamine B solution exhibits a strong and reversible temperaturedependence variation of its fluorescence intensity, it is useful as a temperature detector. The temperature distribution on a microthermodevice was successfully measured from the fluorescence intensity distribution of rhodamine B solution. This method allows temperature distribution measurement with an accuracy of $3{ }^{\circ} \mathrm{C}$ and a spatial resolution of $5.3 \mu \mathrm{m}$. The maximum temperature difference within the circular microheater of our microthermodevice was measured to be $3{ }^{\circ} \mathrm{C}$ [49].

An $\mathrm{F}_{1}$ molecule was attached to a glass surface by infusing $\mathrm{F}_{1}$ contained in buffer in the flow cell. Subsequently, microbeads were attached to the rotary axis of $F_{1}$ by infusing them into the flow cell. Microbeads are necessary because the size of a single $F_{1}$ molecule is too small, around $8 \mathrm{~nm}$ in diameter and $14 \mathrm{~nm}$ in height, to be observed with an optical microscope. The angular velocity change of $F_{1}$ rotation was successfully observed by altering the temperature. Figure $1 \mathrm{~b}$ shows the time course of accumulative revolution of $F_{1}$ while the temperature was switched from room temperature to $50{ }^{\circ} \mathrm{C}$ and back to room temperature. Its angular velocity changed from 1.2 to $4.4 \mathrm{rps}$ and back to $1.7 \mathrm{rps}$. The drawbacks in conventional temperature controlling devices under the microscope such as defocusing caused by thermal expansion of the stage plate were not observed. When the temperature was increased gradually, the velocity of $F_{1}$ increased gradually but stopped at a temperature lower than $50{ }^{\circ} \mathrm{C}$ because the enzyme cannot survive at high temperatures for a long time. To measure the velocity at higher temperatures, the temperature should be altered rapidly, because the enzyme can survive at higher temperatures for a short period, even at high temperatures that would denature it in a steady state. This rapid temperature altering system enabled us to measure the activity of an $\mathrm{F}_{1}$ molecule at temperatures higher than $50{ }^{\circ} \mathrm{C}$. The torque of $\mathrm{F}_{1}$ was calculated by subtracting the effect of water viscosity that causes the drag force on the microbead attached to the rotating axis of $F_{1}$. Consequently, the temperature dependence of the torque of $F_{1}$ could be calculated over a wide temperature range: from room temperature to $70{ }^{\circ} \mathrm{C}$. As a result, the torque seems to increase at higher temperatures, increasing at the rate of $4 \%$ per $10{ }^{\circ} \mathrm{C}$.

MEMS technology enables us to integrate a microheater and a microthermosensor in a small area with ideal design. Also, the microheater and the microthermosensor can be easily electrically connected to a computer and sampled or controlled. Additionally, MEMS technology has massproduction capability that is suitable for biochemical research which requires a large number of experiments for statistical data analysis. This MEMS-based experimental 
tool is versatile in other scientific experiments which require rapid temperature alternation under the microscope.

\section{Rapid temperature alternation by an on-chip thermodevice for enzymatic measurement at high temperatures}

Even though the activity of an enzyme decreases with excess temperature applied over a long time period, we expect the enzyme to stay active by limiting the exposure time $[45,46]$ at temperatures higher than the molecular destruction temperature in a steady state. Enzymatic activity at a high temperature may be measured by momentary heating. However, to raise and reduce the temperature could not be realized by conventional technology in biochemical experiments. There are two problems to solve. One is to change the temperature rapidly and the other is to trap a certain number of molecules within the locally heated space. Arata et al. have developed an on-chip microthermodevice with an integrated microheater and microthermosensor on a glass plate as described in the previous section. Here, the chip was also equipped with a microcontainer array that traps enzymes in the temperaturecontrolled area (Fig. 2). Polydimethylsiloxane (PDMS) was used to form the microchamber because PDMS has the following advantageous characteristics [50]: it has no effect on biomaterials such as proteins and cells [51]; it can be easily patterned into minute structures; it is transparent and allows easy observation of the target inside the structured PDMS; it is compatible with mass production; its adhesion to the flat surface is strong and it is easy to seal with a flat
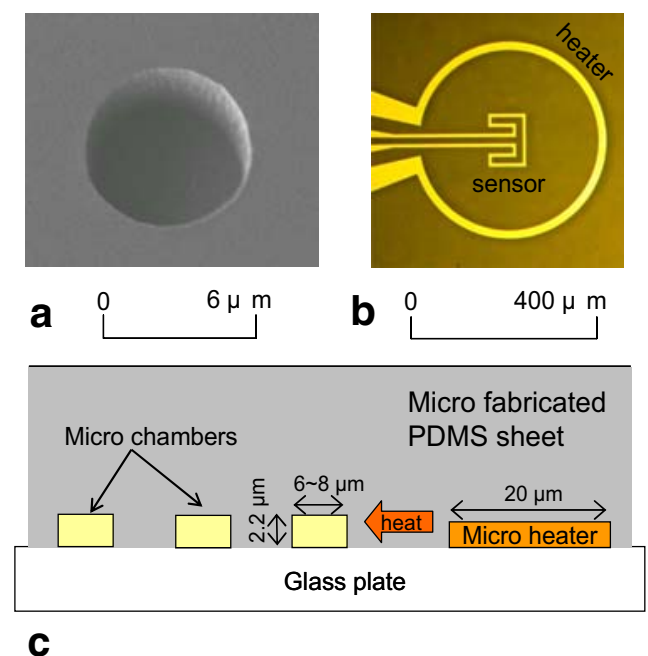

Fig. 2 a Scanning electron microscope image of a patterned polydimethylsiloxane $(P D M S)$ sheet. The 56.0-fL chambers had a standard deviation of 1.63 in volume. b Optical microscope view of an integrated microheater and microthermosensor on a glass plate. $\mathbf{c}$ The temperature-controlled microchamber array. d Activity controlled by temperature pulses with a duty ratio of 1:3 (four pulses in $48 \mathrm{~s}$ ). The substrate. Cylindrical microcontainers with a height of 1.5 $2.2 \mu \mathrm{m}$, a diameter of $1-8 \mu \mathrm{m}$, and a volume of $1-110 \mathrm{fL}$ were manufactured. They were first manufactured to measure the chemical activities of enzymes at the single molecule level by restricting the diffusion of products $[5$, 52]. Here, these containers are utilized for trapping a certain number of molecules within a small volume that is heated.

$\beta$-gal is widely used as a reporter in molecular biology [53]. $\beta$-gal is the enzyme which catalyzes hydrolysis of fluorescein di- $\beta$-D-galactopyranoside (FDG). A buffer solution containing a fluorogenic substrate of $\beta$-gal, FDG, was hydrolyzed to fluorescein. The fluorescence intensity represents the amount of the product produced by $\beta$-gal [5]; therefore, we can measure the activity of $\beta$-gal from the time course of the fluorescence intensity. $\beta$-gal solution was mixed with $400 \mu \mathrm{M}$ FDG in $100 \mathrm{mM}$ phosphate buffer $\mathrm{pH} 7.5$ and contained in a PDMS microchamber. In this experiment, the volume of the microcontainer was $60 \mathrm{fL}$; at a $\beta$-gal concentration of $37 \mathrm{nM}$, each container contained around 1,300 enzymes. Applying $60{ }^{\circ} \mathrm{C}$ heat pulses of $4 \mathrm{~s}$, we successfully increased the activity of the enzyme (Fig. 2d). This indicates that the enzymes remained active and increased their activity over a short period, even at temperatures that cause damage after prolonged exposure; the activity of $\beta$-gal versus temperature shows a peak at around $45-50{ }^{\circ} \mathrm{C}$, a decrease with temperature after the peak and complete cessation above $60{ }^{\circ} \mathrm{C}$ (data shown in Arata et al. [28]). The activity of the enzyme is also controllable by changing the duty ratio of the heating pulses.

This method gives new kinetic information such as the temperature stability of biomolecules and also makes it possible to perform highly sensitive experiments in bio-

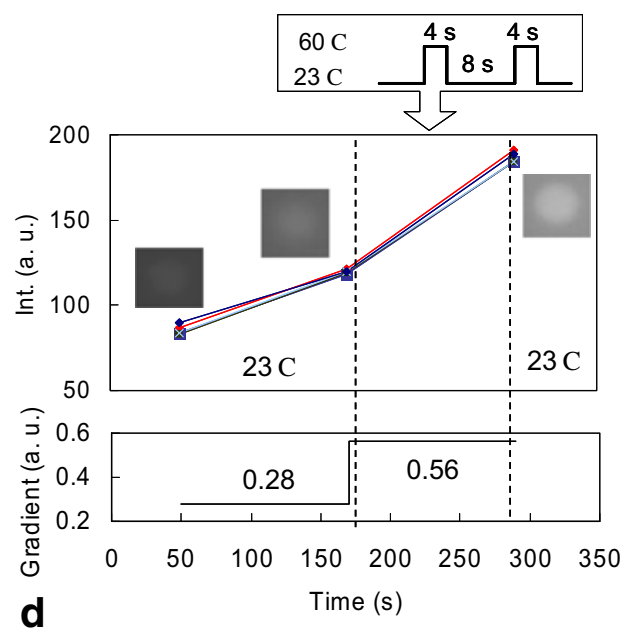

fluorescence intensity increased from 0.28 to 0.56 . The lines follow the intensities of four individual microchambers. Activity was successfully increased, and this indicates that the enzymes remain active and increase their activity over a short period, even at temperatures that cause damage after prolonged exposure. (Reprinted with permission of the American Chemical Society from Arata et al. [28].) 
chemistry, for example, the Arrhienius plot at higher temperatures which does not include the effect of denatured enzymes. As for industrial application, this method is suitable for realizing highly sensitive biosensors. This device can be directly applied to other proteins, cells, and biomaterials for respective heating experiments without any modifications of the device and of the microscope.

\section{Single DNA molecule isolation and trapping in a microfluidic device}

DNA has been widely investigated as it contains genetic information, critical in the understanding of the human body and the future advancement of medical science. In view of this, many researchers have attempted to study DNA through the observation of immobilized DNA molecules. As an example, Harada et al. [54] observed the rotation of single DNA molecule during transcription by RNA polymerase which was immobilized on a cover slip. Immobilization is critical as the first step in realizing a biophysical system used for the manipulation and characterization of biomolecules which move randomly in their natural state. At present, immobilization methods of DNA molecules include (1) chemical binding [55-58], (2) physical stretching and binding [59,60], and (3) electrical stretching and binding $[25,26,61]$. Mixing DNA molecules with a thiol group caused the modified DNA molecules to chemically adhere onto a gold substrate [58]. As an example of a physical binding method, Guan and Lee [60] presented a unique technique for highly ordered arrays of stretched DNA. By using a PDMS sheet with aligned 5 - $\mu \mathrm{m}$-diameter chambers to cover a DNA solution droplet, and then peeling the sheet off slowly, they were able to stretch and immobilize DNA molecules on the peripheral edges of a circular chamber as a result of surface tension and flow pressure. Finally, for electrical binding and stretching, Washizu and Kurosawa [25] proposed an effective way of DNA trapping by dielectrophoresis. In this case, DNA molecules were fully stretched along the electric flux line and immobilized on an aluminum electrode. Microfluidic devices and microfabricated chambers are effective approaches for single molecule experiments. The channel sizes usually used for $\mu$-TAS are in the range from several tens of nanometers to several hundred micrometers. Enclosing molecules in these small areas provides precise handling and good observation for researchers.

Kumemura et al. [29] designed and fabricated a microfluidic device for isolation and immobilization of a single DNA molecule (Fig. 3) which was composed of a PDMS film having wide and narrow channels, and electrodes deposited on a cover slip. The channels were $300 \mu \mathrm{m}$ wide and $4 \mu \mathrm{m}$ deep for the wide channel and $1 \mu \mathrm{m}$ wide and

\section{a}

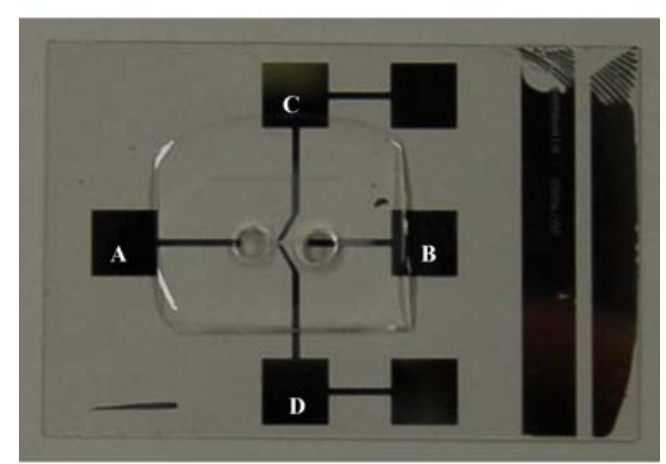

b

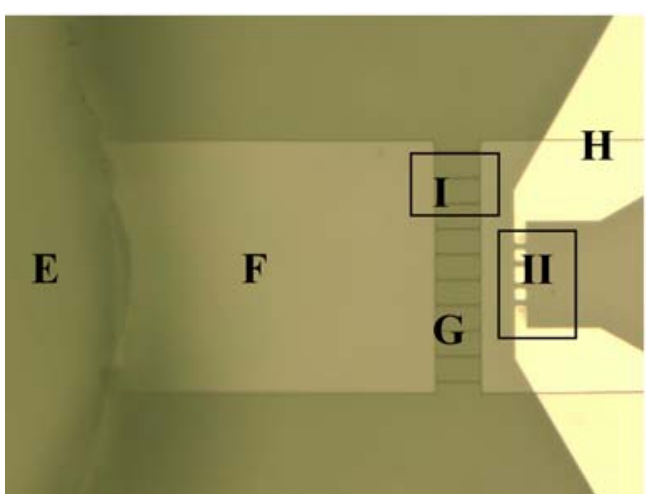

Fig. 3 a A fabricated microdevice. $A$ and $B$ are electrodes for electrophoresis. $C$ and $D$ are electrodes for dielectrophoresis. b Blowup of the microdevice. $E$ is an access hole, $F$ is a wide channel (300 $\mu \mathrm{m}$ wide), $G$ is a narrow channel ( $2 \mu \mathrm{m}$ wide), and $H$ is an electrode for immobilization. (Reproduced with permission from Kumemura et al. [29]. Copyright Wiley-VCH Verlag GmbH \& Co. KGaA.)

$4 \mu \mathrm{m}$ deep for the narrow channel, respectively. In this microdevice, the balance between electrophoresis and electroosmosis in the channel was effectively exploited for DNA molecule isolation. By applying a DC voltage, DNA molecules, which are negatively charged, flow from the anode to the cathode in a microchannel. On the other hand, because the surface of PDMS and the cover slip are negatively charged (silanol group), cations in water which are positioned close to channel walls move from the cathode to the anode. Therefore, electroosmotic flow appeared in the opposite direction to DNA electrophoresis in this case. In the $300-\mu \mathrm{m}$-wide channel, the electrophoretic flow dominated over the effect of electroosmosis. In the narrow channel, the electroosmosis produced significant flow at the entrance of the 1- $\mu \mathrm{m}$-wide microchannel. DNA molecules that reached the entrance of the microchannel were faced with electroosmotic flow in the reverse direction and many of them were prevented from passing through the microchannel (Fig. 4). This efficiency of the molecule separation was estimated by measuring the molecular flux (number of molecules per second) at given locations in the device. The relationship between the molecular flux in the narrow channel and the 
a

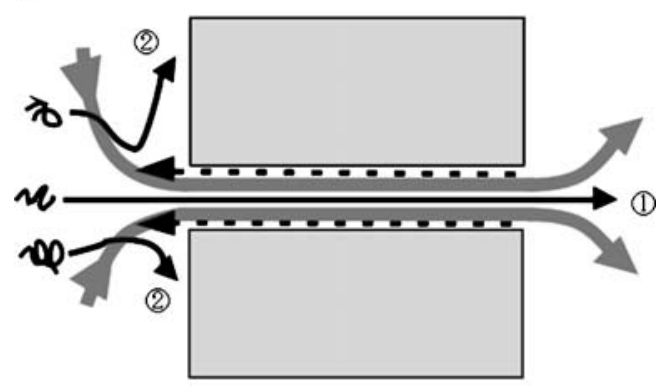

b

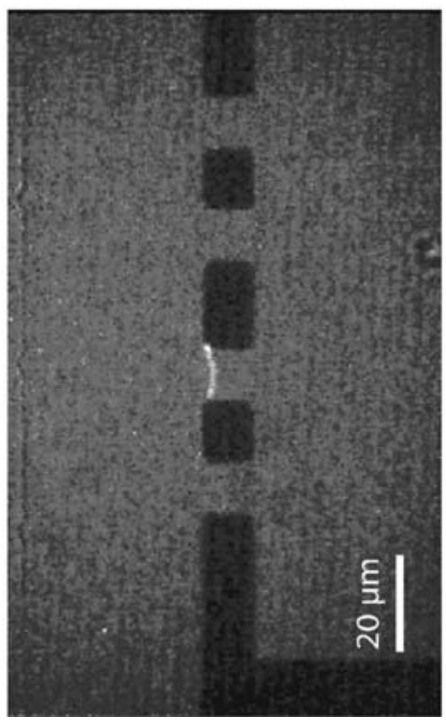

Fig. 4 a The balance between electrophoresis and electroosmosis at the entrance of the narrow channel. The thick solid line shows electrophoresis-induced force line, the dotted line shows electroosmotic flow, and thin solid lines show DNA trajectories. The electrophoresis drift pushes DNA towards the channel entrance. The electroosmotic flows is in the reverse direction and spreads out when exiting the microchannel. This diverging electroosmosis pushes the molecule away from the entrance (case 2). Only the molecule moving in the channel axis can enter (case 1) b The fluorescence view of an immobilized single DNA molecule between fabricated electrodes. (Reproduced with permission from Kumemura et al. [29]. Copyright Wiley-VCH Verlag GmbH \& Co. KGaA.)

applied voltage of electrophoresis was examined and the optimized voltage was obtained for molecule separation. This technique leads to successful single molecule immobilization between electrodes, which is the next step. For immobilization of DNA molecules, electrostatic force (dielectrophoresis) was employed. By applying $900-\mathrm{kHz}$ AC voltage between electrodes positioned at the exit of the narrow channel, a single DNA molecule stretched along the lines of electric force, and was attracted to both edges of the electrodes (Fig. 4). Because the DNA binding on aluminum is nonspecific and permanent $[25,26]$, once the DNA molecule is immobilized on the aluminum electrodes, the stretched molecule provides a stable appropriate template for further biomolecular assay.

\section{Humidity dependence of charge transport through DNA revealed by silicon microtweezers}

DNA has been considered as a possible building block, such as an electric nanowire, for molecular electronic devices. For such an application to nanoelectronics, it is necessary to know the electrical properties of DNA and the physical origin of DNA conductivity. Tran et al. [62] studied the influence of temperature on DNA conductivity and also noted the effect of water. Other groups later discussed the major influence of humidity on DNA conductivity [63-66]. However, the relation between DNA conductivity caused by humidity and the influence of other dimensional parameters such as DNA length or the bundle diameter remained to be determined.

In order to address the question of DNA conductivity and the influence of humidity, Yamahata et al. [67] handled DNA molecules with MEMS-based microtweezers; the

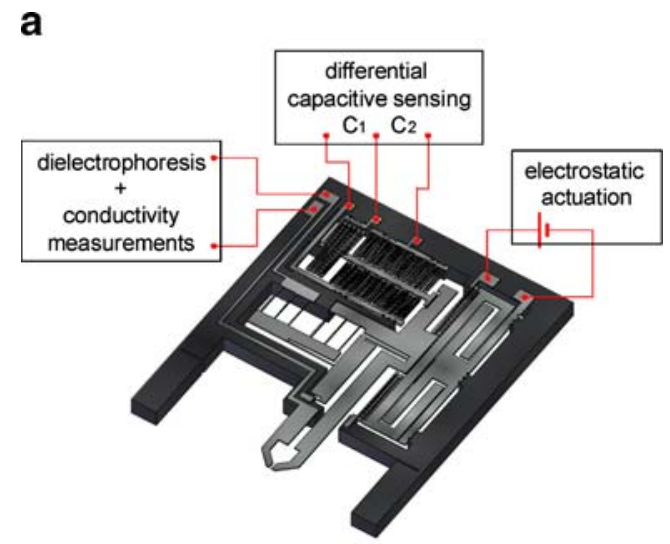

b

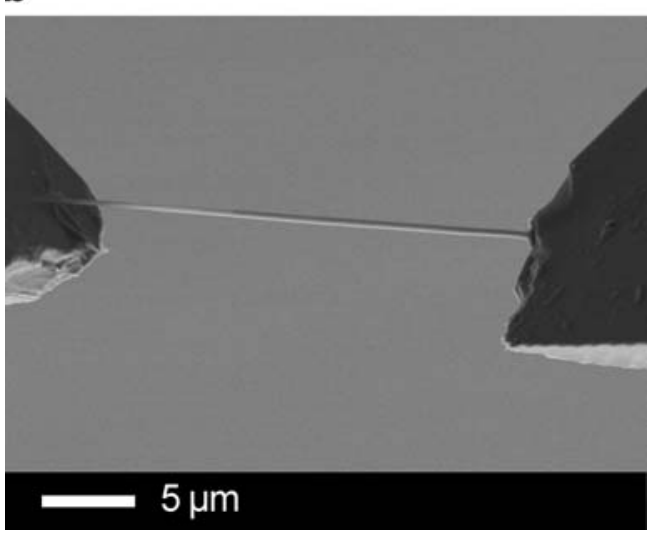

Fig. $53 \mathrm{D}$ view of the microelectromechanical system (MEMS) silicon device and micrograph of a DNA bundle. a The MEMS nanotweezers were fabricated using silicon on insulator technology. The electrostatic actuator, the differential capacitive sensor, and the nanotips are electrically isolated. The displacement, $\Delta l$, of the moving tip results in a change of the variable capacitances $C_{1}$ and $C_{2}$ used for sensing. b A DNA bundle captured between silicon tips coated with aluminum. The bundle has a mean diameter of approximately $380 \mathrm{~nm}$. (Reprinted with permission of the Biophysical Society from Yamahata et al. [30].) 
device enables the straightforward trapping of DNA between aluminum-coated silicon nanotips by AC dielectrophoresis $[68,69]$. The microtweezers were designed to be used for simultaneous mechanical stretching and electrical characterization of the captured DNA bundle (Fig. 5a) [70-72]. With these microtweezers, Yamahata et al. measured the variation of the current $I$ as a function of the applied voltage $U$ (two points measurement method) on DNA bundles (various diameters, $\varphi$ ) in air under a relative humidity of $50-60 \%$ at $25{ }^{\circ} \mathrm{C}$. The diameter of the dried bundles was estimated afterwards by field emission scanning electron microscopy (Fig. 5b). The current $I$ measured for different bundles approximately represents the quasi-linear dependence on $U$. At a constant voltage, the current $I$ shows the quadratic dependence on the diameter $\varphi$ of dried bundles, $I \propto \varphi^{2}$. Yamahata et al. also stretched a thin DNA bundle and simultaneously measured its conductivity (Fig. 6). The relative variation of the current, $\Delta I / I$, as a function of the relative elongation of the bundle, $\Delta l / l$, shows that $\Delta I / I=-\Delta l / l$, indicating that $I \propto 1 / l$ (Fig. 6). These results indicate that the DNA bundle has a quasiohmic behavior, $I=\sigma S U / l$, where $S \propto \varphi^{2}$ is the section of the bundle and $\sigma$ is its conductivity. With an assumption that electron charges $q$ are carried by mobile ions of density $n$, and the kinetics of charge exchanges between ions results in a mobility $\mu$, the conductivity of a DNA bundle can be described as $\sigma=q n \mu$.

Following these experiments, to know the origin of the conductivity of the DNA bundle, Yamahata et al. measured the transient current through a DNA bundle in different humidity conditions. The relative humidity was decreased slowly from

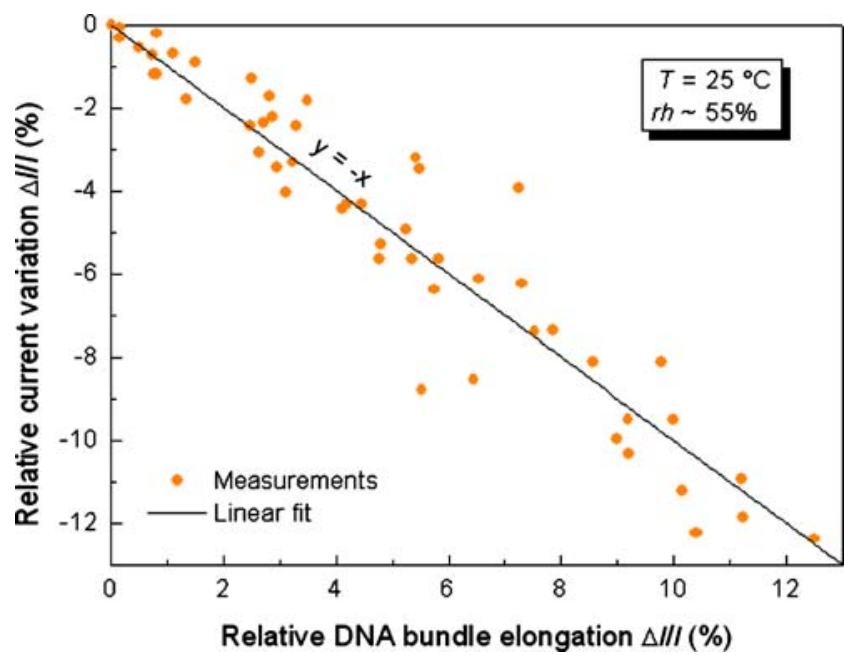

Fig. 6 Effect of the elongation of a DNA bundle on its conductivity. The measurements were performed for a DC voltage of $U=10 \mathrm{~V}$. The data were recorded at $25{ }^{\circ} \mathrm{C}$ for a relative humidity of $50-60 \%$ (typical currents of few tens of picoamperes). The function $y=-x$ is a perfect match with the least-squares linear regression curve calculated over 50 measurements $\left(y=-1.008 x, R^{2}=0.91\right)$. (Reprinted with permission of the Biophysical Society from Yamahata et al. [30].)

\section{a}

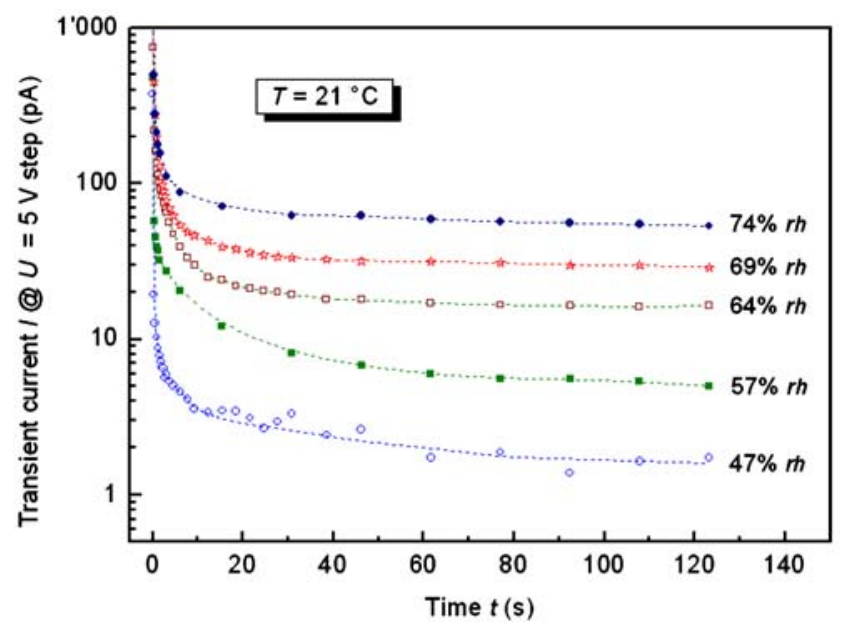

b

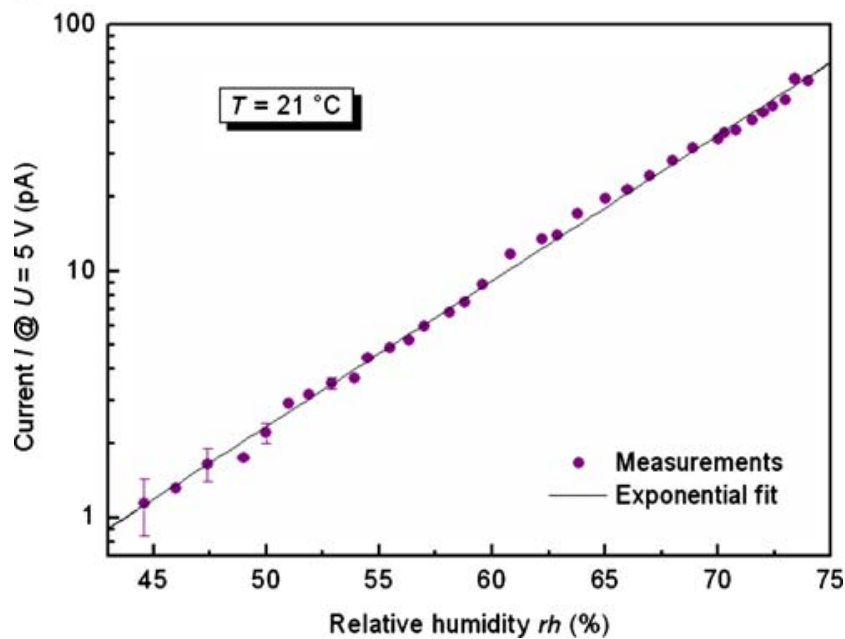

Fig. 7 Effect of the humidity on the transient current and on the conductivity of a DNA bundle. Data recorded at $21{ }^{\circ} \mathrm{C}\left( \pm 1{ }^{\circ} \mathrm{C}\right.$ overall fluctuation) for different humidity levels (relative humidity $\pm 0.2 \%$ for each curve). The relative humidity was decreased from 75 to $45 \%$ in $6 \mathrm{~h}$. a Transient current through a DNA bundle for different humidity conditions. The recording were obtained for a $5-\mathrm{V}$ step. The dotted lines are guides for the eye. $\mathbf{b}$ Exponential decrease of the current with decreasing humidity. The curve is an exponential fit. (Reprinted with permission of the Biophysical Society from Yamahata et al. [30].)

75 to $45 \%$ in $6 \mathrm{~h}$, at a constant temperature of $21^{\circ} \mathrm{C}$. Figure $7 \mathrm{a}$ shows the transient current measured for different values of the relative humidity. In the transient current measurement, three main phenomena resulting in the current decrease can be identified. The authors attributed the first rapid decrease of $I$ with a typical time constant of $\tau_{1} \approx 0.3 \mathrm{~s}$ to electrical capacitance charging in the device, the second one $\left(\tau_{2} \approx 3 \mathrm{~s}\right)$ to the electrical double layer (EDL) charging that takes place at the interface between the electrodes and the moisturized DNA bundle, and the long-term current decrease $\left(\tau_{3} \approx 600 \mathrm{~s}\right)$ to the consumption of carriers by electrolysis [66, 67], respectively. The time constant $\tau_{3}$ is rather large and it is assumed that $\tau_{3} \gg \tau_{1}, \tau_{2}$, so that the current can be 
approximated by an exponential decay with a time constant of $\tau_{3}=l^{2} / \mu U$ after a certain time $t>\tau_{1}, \tau_{2}[73,74]$. With such a timescale in Fig. $7 \mathrm{a}(0 \mathrm{~s} \leq t \leq 120 \mathrm{~s})$, the combined effects of $\tau_{2}$ (which varies with the relative humidity) and $\tau_{3}$ can be seen: For lower humidity levels, because of the reduced concentration of charge carriers, the time required to establish the EDL is longer, resulting in an increase of $\tau_{2}$ (this affects the slope of the curve for $t<60 \mathrm{~s}$ ). For higher humidity levels, mainly the slope due to $\tau_{3}$ can be observed. With the assumption that the decrease in carrier charge was always negligible, the stabilized current (recorded at $t=60 \mathrm{~s}$ in Fig. 7a) at different values of the relative humidity is plotted in Fig. 7b. As previously observed by KleineOstmann et al. [66], the conductivity of the DNA bundle increased exponentially with the relative humidity. The values of $\tau_{3}$, which were extracted from transient measurements recorded over several minutes, did not vary significantly with the relative humidity, indicating that the carrier mobility $\mu$ remains constant with the relative humidity since $\tau_{3}=$ $l^{2} / \mu U$. Therefore, Yamahata et al. concluded that the carrier concentration $n$ is responsible for the current increase with humidity and proposed the following relation for the conductivity: $\sigma \propto \sqrt{N_{\mathrm{w}}} \exp \left(-q^{2} / 2 \varepsilon d R T\right)$, where $N_{\mathrm{w}}$ is the number of water molecules adsorbed per nucleotide, $R$ is the gas constant, $T$ is the absolute temperature, $d$ is the equilibrium separation distance of the charges in the neutral species, and $\varepsilon$ is the permittivity. In this equation, the expression $\exp \left(-q^{2} / 2 \varepsilon d R T\right)$ takes into account the energy needed to separate the charge species during the iongeneration mechanism [63, 75].

The increase of $\sigma$ is mainly caused by the increase of permittivity $\varepsilon$ with the relative humidity $[75,76]$. Several authors attempted to model the charge transfer in the DNAwater complex and considered this system as a heterogeneous dielectric medium consisting of different regions with different dielectric permittivities [77-79]. These authors proposed that DNA in solution has different dielectric zones [79]: the nucleobase, the bases and sugarphosphate backbone, the bound-water zone ( $3 \AA$ thick) adjacent to the surface of the DNA fragment $\left(\varepsilon_{1}\right)$, and the bulk water zone $\left(\varepsilon_{2}\right)$. Yamahata et al. considered that there is space (definitely much more than $3 \AA$ ) between each DNA strand in the bundle: the bundle is a spongelike material rather than a compact assembly of DNA molecules, and the water molecules can penetrate within the bundle. This is consistent with the fact that the current varies linearly with the cross section. Indeed, this dependence shows that the conductivity does not only occur at the surface of the DNA bundle, but also comes from the contribution of each DNA strand inside the bundle. Hence, Yamahata et al. concluded that in their case the global dielectric constant was a function of $\varepsilon_{1}$ and $\varepsilon_{2}$, which both depend on the relative humidity.

\section{Conclusions}

In summary, MEMS-based microsystems have a wide range of possibilities to serve as powerful tools in manipulating and analyzing biomaterials at the molecular level. The miniaturization of experimental tools achieved a fast response speed of a thermodevice which realized rapid temperature alternation in biological experiments. Trapping DNA in a microchannel realized isolation and immobilization on electrodes at the single DNA molecule level. Silicon-based microtweezers enabled us to analyze the humidity dependence of charge transport through DNA. These microdevices with MEMS technologies have additional advantages such as batch process for mass production by a batch process and on-chip integration of multifunctions. This may increase the efficiency of bioexperiments which requires a large number of data and experiments. Furthermore, this approach requires no biological mutations or chemical treatments to the biomaterial itself; hence, it is widely applicable and the compact devices also allow samples to be analyzed at the point of need. We strongly believe that the approach to design and manufacture MEMS devices based on requirements in single molecule measurements might provide breakthroughs and powerful tools in molecular biology.

Acknowledgements The device fabrication processes were conducted using the electron beam lithography equipment donated by ADVANTEST Corporation to the VLSI Design and Education Center (VDEC, University of Tokyo). The work on DNA isolation and trapping was supported by a JST Grant for Advanced Instrumentation "Development of handling and characterization tools for nano objects," while the single DNA observation was supported by a Grant-in-Aid for Scientific Research "Investigation of nano hand-eye system." The work on DNA charge transport using nanotweezers was supported by the Swiss National Science Foundation (grant PBEL2107898), the Japan Society for the Promotion of Science (grant P06373), and a JST Grant for Advanced Instrumentation ("Development of handling and characterization tools for nano objects").

\section{References}

1. Wu MC (1997) Proc IEEE 85(11):1833

2. Kimura M, Toshima K (2003) Sens Actuators A 108:239-243

3. Amato I (1998) Science 282:402

4. Arana LR, Schaevitz SB, Franz AJ, Schmidt MA, Jensen KF (2003) J MEMS 12:600

5. Rondelez Y et al (2005) Nat Biotechnol 23(3):361-365

6. Yamashita K, Sun W, Kakushima K, Fujita H, Toshiyoshi H (2006) J Vac Sci Technol B 24(2):927

7. Isamoto K, Kato K, Morosawa A, Chong C, Fujita H, Toshiyoshi H (2004) IEEE J Select Top Quantum Electron 10(3):570

8. Mi X et al (2006) J Opt A Pure Appl Opt 8:S341-S346

9. Takahashi K, Kwon H-N, Mita M, Saruta K, Lee J-H, Fujita H, Toshiyoshi, H (2007) IEEE J Select Top Quantum Electron 13 (2): 277

10. Toshiyoshi H (2004) JSME Int J Ser B 47(3):439 
11. Yano M, Yamagishi F, Tsuda T (2005) IEEE JSelect Top Quantum Electron 11(2):383-394

12. Yoo SJB (2006) J Lightwave Technol 24(12):4468-4492

13. Manz A, Graber N, Windmer HM (1990) Sens Actuators B 1:244-248

14. Reyes DR, Iossifidis D, Auroux P-A, Manz A (2002) Anal Chem $74: 2623$

15. Auroux P-A, Iossifidis D, Reyes DR, Manz A (2002) Anal Chem 74:2637

16. Ishijima A, Yanagida T (2001) Trends Biochem Sci 26(7):438

17. Noji H, Yasuda R, Yoshida M, Kinoshita K Jr (1997) Nature 386:299

18. Zlatanova J, Leuba SH (2003) Biochem Cell Biol 81:151-159

19. Gosse C, Croquette V (2002) Biophys J 82:3314

20. Ashkin A, Dziedzic JM, Bjorkholm JE, Chu S (1986) Opt Let 11 (5): 288

21. Svoboda K, Block SM (1994) Annu Rev Biophys Biomol Struct 23:247-285

22. Ando T, Kodera N, Takai E, Maruyama D, Saito K, Toda A (2001) Proc Natl Acad Sci USA 98:12469

23. Galletto R, Amitani I, Baskin RJ, Kowalczykowski SC (2006) Nature 443:875

24. Mihajlovic G, Brunet NM, Trbovic J, Xiong P, von Molnar S, Chase PB (2004) Appl Phys Lett 85:1060

25. Washizu M, Kurosawa O (1990) IEEE Trans Ind Appl 26(6):1165

26. Washizu M, Kurosawa O, Arai I, Suzuki S, Shimamoto N (1995) IEEE Trans Ind Appl 31(3):447

27. Arata HF, Noji N, Fujita H (2006) Appl Phys Lett 88:083902

28. Arata HF, Rondelez Y, Noji H, Fujita H (2005) Anal Chem 77 (15):4810-4814

29. Kumemura M, Collard D, Yamahata C, Sakaki N, Hashiguchi G, Fujita H (2007) ChemPhysChem 8:1875-1880

30. Yamahata C, Collard D, Takekawa T, Kumemura M, Hashiguchi G, Fujita H (2008) Biophys J 94:63-70

31. Schliwa M (ed) (2003) Molecular motors. Wiley-VCH, Weinheim

32. Schnitzer MJ, Block SM (1997) Nature 388:386-390

33. Mazumdar M, Mikami A, Gee MA, Vallee RP (1996) Proc Natl Acad Sci USA 93:6552-6556

34. Kitamura K, Tokunaga M, Iwane AH, Yanagida TA (1999) Nature 397:129-134

35. Lohman T, Thorn K, Vale RD (1998) Cell 93:9-12

36. Wang M, Schnitzer M, Yin H, Landick R, Gelles J, Block S (1998) Science 282:902-907

37. Muramoto K, Kawagishi I, Kudo S, Magariyama Y, Imae Y, Homma M (1995) J Mol Biol 251(1):50-58

38. Noji H, Hasler K, Junge W, Kinosita K Jr, Yoshida M, Engelbrecht S (1999) Biochem Biophys Res Commun 260:579-599

39. Yasuda R et al (1998) Cell 93:1117-1124

40. Yasuda R et al (2001) Nature 410:898-904

41. Kato-Yamada Y et al (1998) J Biol Chem 273(31):19375-19377

42. Hess H, Clemmens J, Qin D, Howard J, Vogel V (2001) Nano Lett $1: 235$

43. Tsuda Y, Mashimo T, Yoshiya I, Kaseda K, Harada Y, Yanagida T (1996) Biophys J 71:2733

44. Liu H, Schmidt JJ, Bachand GD, Rizk SS, Looger LL, Hellinga HW, Montemagno CD (2002) Nat Mater 1:173-177

45. Martin J, Horwich AL, Hartl FU (1992) Science 258(5084):995-998

46. Sewell BT, Best RB, Chen S, Roseman AM, Farr GW, Horwich AL, Saibil HR (2004) Nat Struct Mol Biol 11(11):1128-1133

47. Tanaka Y, Slyadnev MN, Hibara A, Tokeshi M, Kitamori T (2000) J Chromatogr A 894:45-51

48. Slyadnev MN, Tanaka Y, Tokeshi M, Kitamori T (2001) Anal Chem 73(16):4037-4044
49. Arata HF, Low P, Ishizuka K, Bergaud C, Kim B, Noji H, Fujita H (2006) Sens Actuators B 117:339-345

50. McDonald JC, Whitesides GM (2002) Acc Chem Res 35(7): 491-499

51. Baltussen E, Sandra P, David F, Hanssen HG, Cramers C (1999) Anal Chem 71:5213-5216

52. Rondelez Y, Tresset G, Nakashima T, Kato, Fujita H, Takeuchi S, Noji H (2005) Nature 433:773-777

53. Alam J, Cook JL (1990) Anal Biochem 188:245-254

54. Harada Y, Ohara O, Takatsuki A, Itoh H, Shimamoto N, Kinoshita JK (2001) Nature 409:113-115

55. Bonanni A, Pividori MI, Valle MD (2007) Anal Bioanal Chem 389:851-861

56. Riccardi CS, Dahmouche K, Santilli CV, Costa PI, Yamanaka H (2006) Talanta 70:637-643

57. Wang Z, Wilkop T, Xu D, Dong Y, Ma G, Quan Cheng Q (2007) Anal Bioanal Chem 389:819-825

58. Germishuizen WA, Walti C, Tosch P, Wirtz R, Pepper M, Davies AG, Middelberg APJ (2003) IEE Proc Nanobiotechnol 150:54-58

59. Michalet X, Ekong R, Fougerousse F, Rousseaux S, Schurra C, Hornigold N, van Slegtenhorst M, Wolfe J, Povey S, Beckmann JS, Bensimon A (1997) Science 277:1518-1523

60. Guan J, Lee LM (2005) Proc Natl Acad Sci USA 102:1832118325

61. Dewarrat F, Calame M, Schonenberger C (2002) Single Mol 4:189-193

62. Tran P, Alavi B, Gruner G (2000) Phys Rev Lett 85:1564-1567

63. Ha DH, Nham H, Yoo KH, So HM, Lee HY, Kawai T (2002) Chem Phys Lett 355:405-409

64. Armitage NP, Briman M, Grüner G (2004) Physi Status Solidi B 241:69-75

65. Tuukkanen S, Kuzyk A, Toppari J, Hytönen VP, Ihalainen T, Torma P (2005) Appl Phys Lett 87:183102/1-183102/3

66. Kleine-Ostmann T, Jördens C, Baaske K, Weimann T, de Angelis MH, Koch M (2006) Appl Phys Lett 88:102102/1-102102/3

67. Yamahata C, Collard D, Takekawa T, Kumemura M, Hashiguchi G, Fujita H ( 2007) Biophys J. DOI 10.1529/biophysj.107.115980

68. Hosogi M, Hashiguchi G, Haga M, Yonezawa T, Kakushima K, Fujita H (2005) Jpn J Appl Phys Part 2 44:L955-L957

69. Hashiguchi G, Goda T, Hosogi M, Hirano K, Kaji N, Baba Y, Kakushima K, Fujita H (2003) Anal Chem 75:4347-4350

70. Collard D, Yamahata C, Legrand B, Takekawa T, Kumemura M, Sakaki N, Hashiguchi G, Fujita H (2007) IEEJ Trans Electr Electron Eng 2:262-271. DOI 10.1002/tee.20154

71. Yamahata C, Takekawa T, Ayano K, Hosogi M, Kumemura M, Legrand B, Collard D, Hashiguchi G, Fujita H (2006): In: Proceedings of the IEEE international conference on microtechnologies in medicine and biology, Okinawa, Japan, pp 123126. DOI 10.1109/MMB.2006.251507

72. Yamahata C, Takekawa T, Kumemura M, Hosogi M, Hashiguchi G, Collard D, Fujita H (2007) In: Technical digest of Transducers '07, the 14th International conference on solid-state sensors, actuators and microsystems, Lyons, France, vol 1, pp 395-398

73. Watanabe M, Rikukawa M, Sanui K, Ogata N (1985) J Appl Phys 58:736-740

74. Nilsson M (2006) Phys Status Solidi C 3:251-254

75. Anderson JH, Parks GA (1968) J Phys Chem 72:3662-3668

76. Yeh Y.C, Tseng TY (1989) J Mater Sci 24:2739-2745

77. Yang LQ, Weerasinghe S, Smith PE, Pettitt BM (1995) Biophys J 69:1519-1527

78. Tavernier H, Fayer M (2000) J Phys Chem B 104:11541-11550

79. Siriwong K, Voityuk AA, Newton MD, Rösch N (2003) J Phys Chem B 107:2595-2601 